\title{
Augmentation of antibody-dependent cellular cytotoxicity with defucosylated monoclonal antibodies in patients with GI-tract cancer
}

\author{
TAKAHIRO NAKAJIMA ${ }^{1}$, HIROKAZU OKAYAMA ${ }^{1}$, MAI ASHIZAWA ${ }^{1}$, \\ MASARU NODA $^{2}$, KEITA AOTO ${ }^{1}$, MOTONOBU SAITO ${ }^{1}$, TOMOYUKI MONMA ${ }^{1}$, \\ SHINJI OHKI ${ }^{1}$, MASAHIKO SHIBATA ${ }^{1}$, SEIICHI TAKENOSHITA ${ }^{1}$ and KOJI KONO ${ }^{1}$ \\ Departments of ${ }^{1}$ Gastrointestinal Tract Surgery and ${ }^{2}$ Breast Surgery, \\ Fukushima Medical University, Fukushima 960-1295, Japan
}

Received July 25, 2017; Accepted November 6, 2017

DOI: $10.3892 / \mathrm{ol} .2017 .7556$

\begin{abstract}
Enhancement of antibody-dependent cellular cytotoxicity (ADCC) with some modalities may be a promising approach to enhance the efficacy of therapeutic monoclonal antibodies (mAbs). It has previously been demonstrated that the removal of fucose from antibody oligosaccharides (defucosylation) leads to augmentation of ADCC activity. To establish clinically relevant evidence of this procedure, the present study evaluated trastuzumab- and cetuximab-mediated ADCC by comparing defucosylated $\mathrm{mAbs}$ with conventional $\mathrm{mAbs}$ using peripheral blood mononuclear cells (PBMCs). PBMCs were isolated from 20 patients with gastrointestinal tract cancer and 10 healthy volunteers. ADCCs were measured using PBMCs as effector cells and two gastric cancer cell lines as target cells. ADCCs were significantly enhanced with defucosylated mAbs compared with conventional mAbs using PBMC from the healthy donors and patients with cancer. The results confirmed that the cetuximab- and trastuzumab-mediated ADCCs in advanced disease were impaired in comparison to those in early disease or healthy individuals. However, when the defucosylated mAbs were used instead of the conventional mAbs, the ADCC activities in the advanced cases were almost comparable with those in early disease or healthy individuals. Furthermore, the expression of ADCC associated molecules were modified toward immunosuppressive status with a mitogen-activated protein kinase inhibitor in vitro, the conventional cetuximab- and trastuzumab-mediated ADCC was downregulated, and the defucosylated mAbs overcome the downregulation of ADCC. In conclusion, defucosylated
\end{abstract}

Correspondence to: Professor Koji Kono, Department of Gastrointestinal Tract Surgery, Fukushima Medical University, 1 Hikarigaoka, Fukushima 960-1295, Japan

E-mail: kojikono@fmu.ac.jp

Key words: antibody-dependent cellular cytotoxicity, defucosylation, trastuzumab, cetuximab, gastrointestinal tract cancer therapeutic mAbs may enhance ADCC activities in patients with cancer, which may lead to more effective anti-cancer treatments.

\section{Introduction}

Although improvement of systemic chemotherapy has been demonstrated in gastrointestinal (GI) tract cancer including gastric and colon cancer, the overall survival rate in patients with advanced stage is still poor (1). Therefore, it would be desirable to develop molecular target therapy more efficiently in GI-tract cancer. For example, for the human epidermal growth factor receptor (EGFR) related 2 (HER2)-overexpressing gastric cancer, Trastuzumab in combination with chemotherapy vs. chemotherapy alone for treatment of HER2-positive advanced gastric cancer (ToGA) trial concluded that anti-HER2 monoclonal antibody (trastuzumab) plus chemotherapy is a standard treatment option, in which trastuzumab plus chemotherapy showed better survival in comparison to chemotherapy alone (2).

It is generally accepted that trastuzumab can act on gastric cancer cells through both anti-proliferative function directly to cancer cells and antibody-dependent cellular cytotoxicity (ADCC) activity via immune cells $(3,4)$. It has been reported that Trastuzumab-mediated ADCC can be influenced by several factors including single nucleotide polymorphisms $(S N P S)$ in the $\mathrm{Fc}$ gamma receptor $(\mathrm{Fc} \gamma \mathrm{R})$ genes (5-7) or natural killer (NK) cell dysfunction (8). In fact, the SNPs can alter the $\mathrm{Fc} \gamma \mathrm{R}$ binding affinity to the therapeutic monoclonal antibodies (mAbs) and consequently resulted in impairment of the ADCC activity. Of importance, a clinical trial showed that therapeutic efficacy of trastuzumab against HER2-positive breast cancer was significantly different between patients with and without certain SNPs in the Fc $\gamma \mathrm{R}$ genes (9). Furthermore, the same observation was also confirmed in colorectal cancer treated with anti-EGFR antibody, cetuximab (10). These results strongly suggest that enhancement of ADCC with some modalities would be a promising approach to enhance the efficacy of therapeutic mAbs. 
It has been shown that removal of fucose from antibody oligosaccharides attached to $\mathrm{Asn}^{297}$ of the heavy chain (defucosylation) significantly enhanced $\mathrm{Fc} \gamma \mathrm{R}$ binding affinity between $\mathrm{Fc} \gamma \mathrm{R}$ on NK cells and the mAbs, in comparison to that of conventional antibody, leading to augmentation of ADCC activity (11-15). Thus, the defucosylation technology could be one of the most powerful approaches to enhance clinical efficacy of therapeutic mAbs. There is, however, still limited information describing the clinical usefulness of the defucosylated therapeutic antibody on the ADCC, except for one report showing that the use of the defucosylated antibodies may improve the therapeutic effects of trastuzumab for breast cancer patients (16). Thus, it is necessary to draw solid conclusion for the effectiveness of the defucosylated antibody in cancer patients or immunosuppressive state. In the present study, using PBMCs from GI tract cancer patients and healthy donors, we evaluated trastuzumab- and cetuximab-mediated ADCC by comparing the defucosylated mAbs with conventional mAbs. This is the first report using PBMCs from patients of GI tract cancer. In addition, when ADCC-related molecules are modulated by mitogen-activated protein kinase (MAPK) inhibitors, the trastuzumab- and cetuximab-mediated ADCC were also evaluated.

\section{Materials and methods}

Preparation of human effector cells. Twenty patients with histologically diagnosed GI tract cancer, who were treated at Fukushima Medical University Hospital (Fukushima, Japan) from February to August in 2016, were enrolled. PBMCs were isolated from esophageal $(n=4)$, gastric $(n=9)$, and colon cancer patients $(n=7)$, and healthy individuals $(n=10,34.8 \pm 7.8$ years old, Male: Female=9:1). PBMCs were separated by lymphocyte separation solution (Lymphoprep ${ }^{\mathrm{TM}}$, Cosmo Bio Company) with density gradient. None of the patients received radiotherapy, chemotherapy, surgery, or other medical interventions before this study. Patients' characteristics are shown in Table I. This study was approved by the ethical committee of Fukushima Medical University (approval no. 2353), and informed consent for blood donations was obtained for all individuals.

Cell lines. MKN-7 (HER-2 overexpressing gastric cancer cell lines; cat. no. JCRB1025) and K562 (myelogenous leukemia cell lines; cat. no. JCRB0019) were purchased from the Japanese Collection of Research Bioresources (Osaka, Japan). MKN-28 (EGFR overexpressing gastric cancer cell line) was obtained from the American Type Culture Collection (Rockville, MD, USA). The MKN28 cell line has previously been reported to be a mixed gastric cancer type, with MKN74 (an EGFR overexpressing cancer cell line) contamination (17). However, this contamination is not thought to have affected the results of the present study as MKN28 and MKN74 share similar characteristics in terms of EGFR overexpression, as described previously (18). All cell lines were maintained in RPMI-1640 (Sigma-Aldrich; Merck KGaA, Darmstadt, Germany) with $10 \%$ fetal bovine serum (Nichirei Biosciences, Inc., Tokyo, Japan) and $1 \%$ penicillin/streptomycin (Nichirei Biosciences, Inc.) at $37^{\circ} \mathrm{C}$ and $5 \% \mathrm{CO}_{2}$.

Antibodies. Anti-HER-2 monoclonal antibody trastuzumab and anti-human EGFR antibody cetuximab were used as clinical
Table I. Characteristics of the patients $(n=20)$.

\begin{tabular}{lc}
\hline Characteristic & Number of patients (n) \\
\hline Age, years (median, range) & $54-80(65)$ \\
Male:female & $17: 3$ \\
Location of carcinoma & \\
Esophagus & 4 \\
Stomach & 9 \\
Colon & 7 \\
Clinical stage (TNM classification) & \\
0 & 2 \\
1 & 6 \\
2 & 2 \\
3 & 2 \\
4 & 2 \\
\hline
\end{tabular}

TNM, tumor-node-metastasis classification of malignant tumors.

grade products, and their defucosylated version were provided by Kyowa Hakko Kirin Co., Ltd. (Tokyo, Japan), which were designed according to the known amino acid sequences $(19,20)$ and produced with parent or $\alpha-1,6$-fucosyltransferase knockout Chinese hamster ovary cells (21).

Antibody-dependent cellular cytotoxicity (ADCC) assay. Cytotoxicity was determined by the lactate dehydrogenase (LDH) release assay using PBMCs as effector cells and either MKN-7 cells or MKN-28 cells as target cells. Briefly, target cells $\left(5 \times 10^{3}\right.$ per well) were distributed into 96 -well U-bottomed plates and pre-incubated with mAbs for $1.5 \mathrm{~h}$ at $37^{\circ} \mathrm{C}, 5 \% \mathrm{CO}_{2}$. Then, effector cells were added at indicated doses and incubated for $7 \mathrm{~h}$. Assays were performed in triplicate with or without antibodies. The supernatant LDH activity was measured using a nonradioactive cytotoxicity assay kit (Cytotoxicity Detection kit ${ }^{\text {PLus; }}$, Roche Diagnostics, Basel, Switzerland) and was measured at $490 \mathrm{~nm}$ excitation and $650 \mathrm{~nm}$ emission wavelengths using spectrometer. Percentage cytotoxicity was calculated according to the formula: Cytotoxicity $(\%)=100 \times$ (Experimental release-Spontaneous release)/(Maximum release-Spontaneous release). The maximum release was prepared with target cells lysed with the lysis solution. In the several pre-test run, the spontaneous release of effector cells was approximately zero. Net ADCC was calculated according to the formula: net ADCC $(\%)=A D C C$ activities (\%)-antibody-independent cellular cytotoxicity (AICC; \%), where AICC is the nonspecific cytotoxicity in the absence of antibodies.

Cell treatment with MAPK signal inhibitors. Tumor cells were cultured in a 6-well plate and exposed to the MAPK signal inhibitor, PD0325901 (Selleck Chemicals, Houston, TX, USA) as indicated in our previous report (22). Then, cytotoxicity assays were performed after $48 \mathrm{~h}$ of incubation.

Statistical analysis. Date comparing differences between two groups assessed using unpaired Student's t-test and two way 

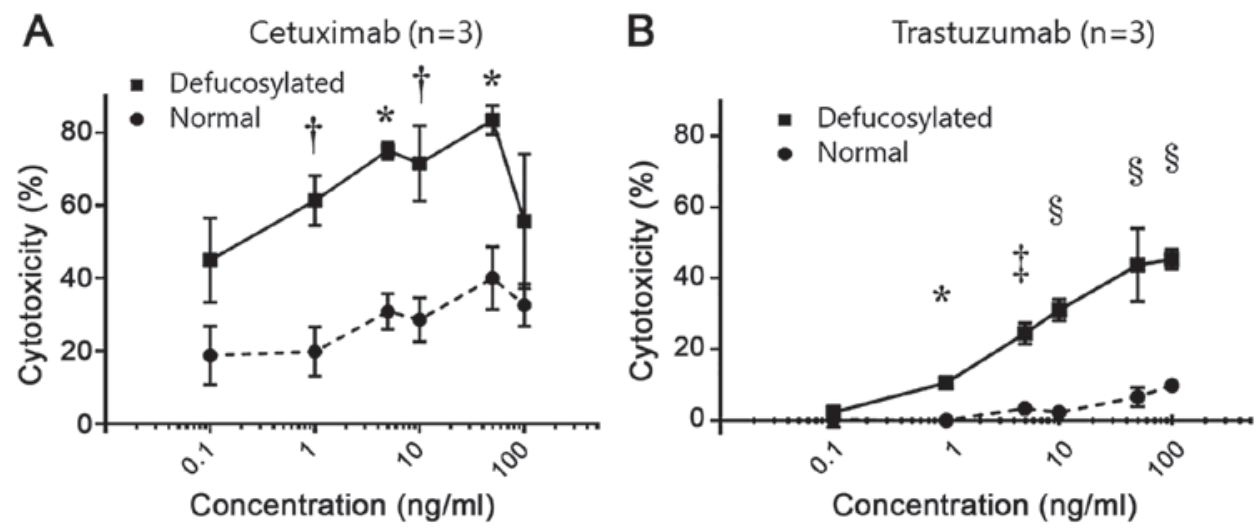

Figure 1. Optimal concentration of defucosylated mAbs. (A) Cetuximab-mediated and (B) trastuzumab-mediated antibody-dependent cellular cytotoxicity were evaluated in various concentrations of conventional (Normal) and defucosylated mAbs using peripheral blood mononuclear cells from healthy donors $(\mathrm{n}=3)$. Data are presented as the mean \pm standard deviation of the cytotoxicity $(\%)$ in triplicates at effector: target ratio of $40: 1 .{ }^{*} \mathrm{P}<0.05,{ }^{\circ} \mathrm{P}<0.005,{ }^{{ }^{\prime}} \mathrm{P}<0.0005$ and ${ }^{\S} \mathrm{P}<0.00005$ vs. Normal. mAbs, monoclonal antibodies.

ANOVA. $\mathrm{P}<0.05$ was considered to indicate a statistically significant difference.

\section{Results}

Optimal condition of defucosylated therapeutic $m A b s$ for ADCC activity. Cetuximab-mediated and trastuzumab-mediated ADCCs were evaluated in various concentrations of conventional and defucosylated mAbs using healthy donor's PBMCs ( $\mathrm{n}=3$, Fig. $1 \mathrm{~A}$ and $\mathrm{B})$. The EGFR-positive MKN28 gastric cancer cell line was used for cetuximab-mediated ADCC and the HER2-positive MKN7 gastric cancer was used for trastuzumab-mediated ADCCs, and the overexpression of EGFR or HER2 on tumor cells were repeatedly confirmed by flow cytometry (data not shown).

As shown in Fig. 1A, defucosylated cetuximab-mediated ADCCs at effector: Target ratio of 40:1 were significantly higher than conventional cetuximab-mediated ADCCs in each concentration. We observed a dose-dependent increase from 0.1 to $50 \mathrm{ng} / \mathrm{ml}$ and thereafter the ADCC leaded to a drop in the present experimental condition, consistent with the previous report (21). Therefore, $50 \mathrm{ng} / \mathrm{ml}$ of defucosylated and conventional cetuximab were used for subsequent experiments as optimal doses.

Also, the same tendency was observed in trastuzumab-mediated ADCCs (Fig. 1B) and $50 \mathrm{ng} / \mathrm{ml}$ of defucosylated and conventional trastuzumab were used for subsequent experiments as optimal doses.

Augmented ADCC by defucosylated cetuximab and trastuzumab. ADCC activities mediated by either conventional or defucosylated mAbs using PBMCs from cancer patients $(n=20)$ and healthy volunteers $(n=10)$ were evaluated. The patient's background is shown in Table I. In order to confirm the condition of PBMCs as effector cells, NK cell activities targeted for K562 were also evaluated in parallel to the ADCC assay and we confirmed condition of NK status in each experiment (Fig. 2).

As shown in Fig. 3A, the defucosylated cetuximab-mediated ADCCs were markedly enhanced in comparison to conventional cetuximab-mediated ADCCs in healthy donor's

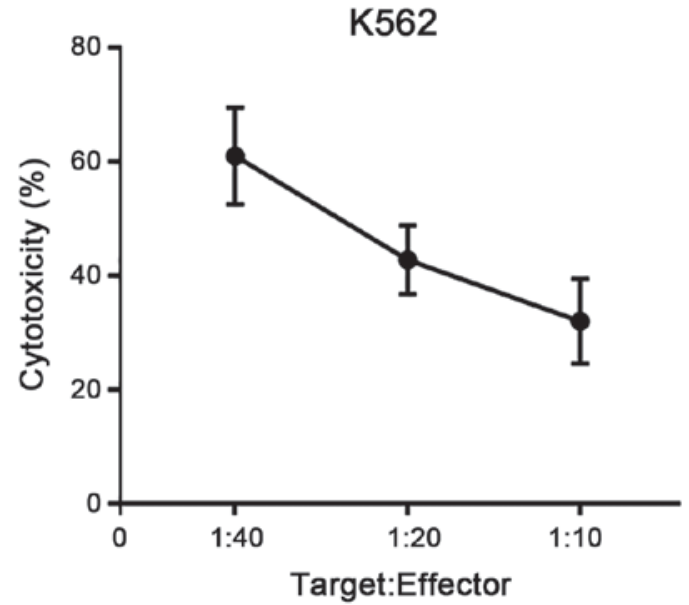

Figure 2. Confirmation of the optimal NK cell activities. NK cell activities targeted for K562 in the peripheral blood mononuclear cells of healthy individuals $(n=10)$ were evaluated in parallel to the antibody-dependent cellular cytotoxicity assay; the optimal NK activities under these conditions were identified. Data are presented as the mean \pm standard deviation. NK, natural killer.

PBMCs. For example, the defucosylated and conventional cetuximab-mediated ADCC at 40:1 ratio were 58.9 \pm 7.5 and $33.5 \pm 3.9 \%$, respectively. Similar observation was also confirmed using the PBMCs from cancer patients (Fig. 3B), in which the defucosylated and conventional trastuzumab-mediated ADCC at 40:1 ratio were 52.9 \pm 4.0 and $32.0 \pm 2.8 \%$, respectively.

Also, the enhancement by defucosylated mAbs was confirmed in the trastuzumab-mediated ADCCs in both healthy donors and cancer patients (Fig. 3C and D).

Taken together, the defucosylated therapeutic mAbs can enhance the ADCC activities in comparison to the conventional mAbs using PBMCs from both healthy donors and cancer patients.

Defucosylated cetuximab- and trastuzumab-mediated ADCC in advanced cancer cases. Based on the UICC-TNM classification, we classified the cancer patients into advanced disease corresponded to stage III and IV, or into early disease 


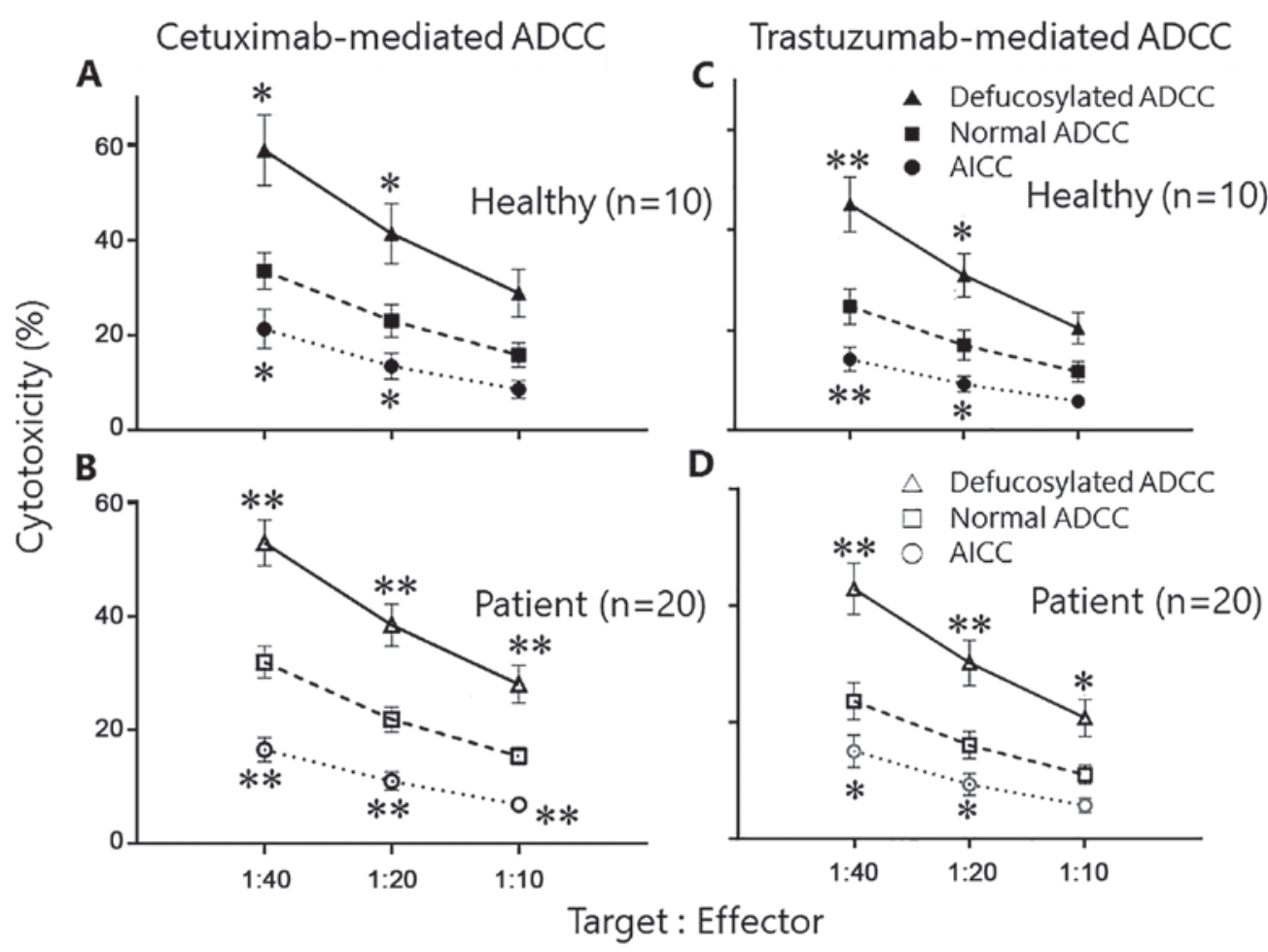

Figure 3. Augmented ADCC by defucosylated cetuximab and trastuzumab. ADCC activities mediated by either conventional or defucosylated monoclonal antibodies using peripheral blood mononuclear cells from (B and D) cancer patients $(n=20)$ and $(A$ and $C)$ healthy volunteers $(n=10)$. Data are presented as the mean \pm standard deviation. ${ }^{*} \mathrm{P}<0.05$ and ${ }^{* *} \mathrm{P}<0.005$ vs. Normal ADCC. ADCC, antibody-dependent cellular cytotoxicity; AICC, the nonspecific cytotoxicity in the absence of antibodies.

corresponded to stage $0, \mathrm{I}$, and II. It has been already reported that the ADCC activities in advanced cancer patients were impaired due to several mechanisms, including NK cell dysfunction or immunosuppressive factors $(8,23,24)$. As expected, we confirmed that the cetuximab-mediated and trastuzumab-mediated ADCCs in advanced disease were impaired in comparison to those in early disease or healthy individuals (Fig. 4). However, when the defucosylated mAbs were used instead of the conventional mAbs, the ADCC activities in the advanced cases were almost comparable to those in early disease or healthy individuals (Fig. 4) and this observation was confirmed in both defucosylated cetuximab and trastuzumab.

Thus, the defucosylated therapeutic mAbs can rescue the impaired ADCC in advanced disease.

ADCC by defucosylated cetuximab and trastuzumab when treated with MAPK inhibitors. In order to further investigate defucosylated therapeutic mAbs-mediated ADCC, we modified the expression of natural killer group 2 member $\mathrm{D}$ receptor (NKG2D) ligand and major histocompatibility complex (MHC) class I on tumor cells by MAPK inhibitors as indicated in our previous report (22). It is generally accepted that NK cells can react with tumor cells through the balance of inhibitory and stimulatory signals. The interaction between the killer immunoglobulin-like receptor family on NK cells and MHC class I molecules results in inhibitory signals, whereas activating signals by NKG2D ligands expressed on targets induce stimulatory signals leading to target cell killing (25-27). We have shown that treatment of target tumor cells with MAPK inhibitors can decrease ADCC activities through upregulation of MHC class I and down-regulation of NKG2D ligands such as MICA/B (22). As expected, conventional cetuximab- and trastuzumab-mediated ADCC was significantly decreased, when target tumor cells were pre-treated with the MAPK inhibitor (Fig. 5). However, when the defucosylated mAbs were used instead, ADCC activities did not alter even if the target cells were pre-treated with the MAPK inhibitor. In the current experiment condition, MAPK inhibitors do not have any direct anti-proliferative effect on target cancer cells (data not shown).

Taken together, defucosylated therapeutic mAbs can efficiently enhance ADCC activities, even if the NKG2D ligand and MHC class I expression on tumor cells, which are corresponding to immune suppressive status, were modified.

\section{Discussion}

The present study provide an important finding relevant to clinical cancer treatment with therapeutic mAbs. First, we showed the augmentation of ADCC by defucosylated therapeutic mAbs using PBMCs from healthy donors and cancer patients. Second, although the ADCC activities were impaired in advanced disease, the defucosylated mAbs can restore the impaired ADCC to the levels of healthy individuals. Finally, the defucosylated therapeutic mAbs can enhance ADCC activities even if the NKG2D ligand and MHC class I expression on tumor cells were modified to induce immunosuppressive environment.

There is accumulating evidence that ADCC is an important antitumor mechanism when the therapeutic mAbs showed the clinical benefit (28-32), and augmentation of ADCC will be 

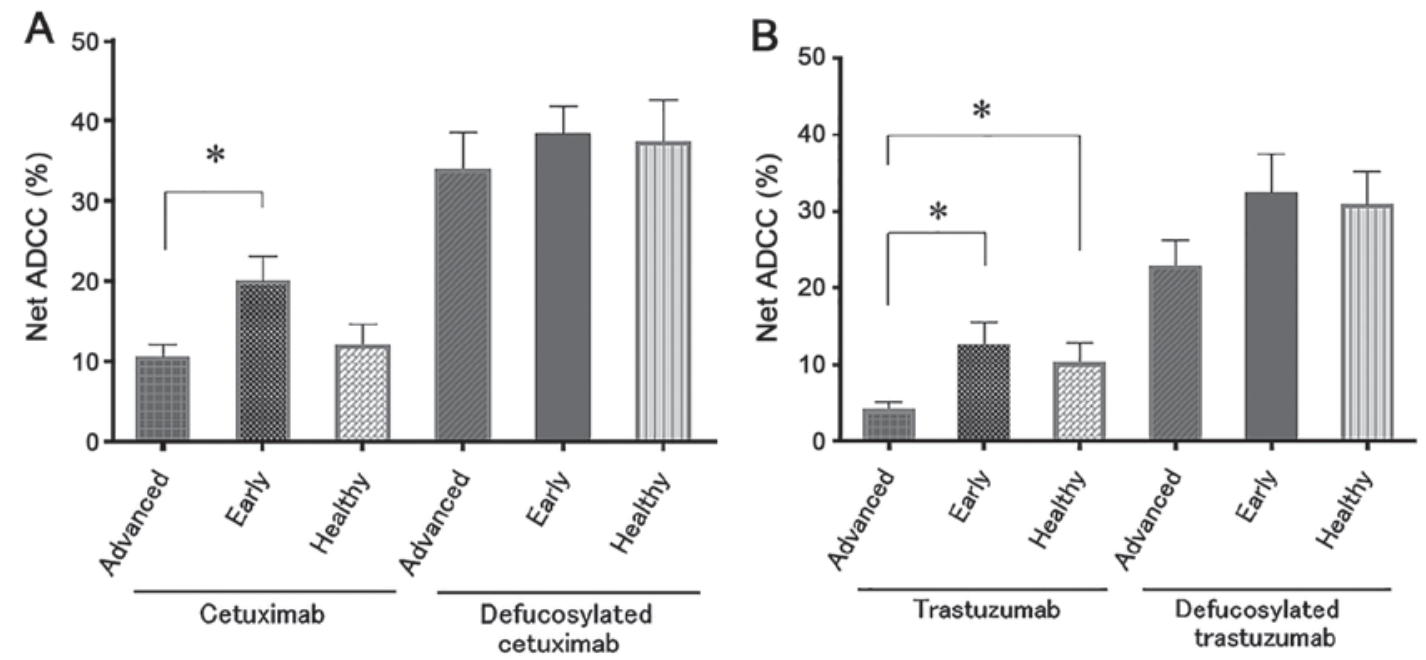

Figure 4. Defucosylated cetuximab- and trastuzumab-mediated ADCC in advanced cancer cases. Net ADCC in advanced disease (n=10), early disease $(\mathrm{n}=10)$ and healthy individuals $(\mathrm{n}=10)$ were evaluated in $(\mathrm{A})$ cetuximab-mediated and $(\mathrm{B})$ trastuzumab-mediated ADCCs. Net ADCC $(\%)=\mathrm{ADCC}$ activities (\%)-antibody-independent cellular cytotoxicity (\%). Data are presented as the mean \pm standard deviation. "P $<0.05$, as indicated. ADCC, antibody-dependent cellular cytotoxicity.



Figure 5. ADCC is induced by defucosylated cetuximab and trastuzumab when treated with MAPK inhibitors. Target tumor cells were pretreated with a MAPK inhibitor (PD0325901), and (A) cetuximab-mediated ADCCs or (B) trastuzumab-mediated ADCCs were evaluated in peripheral blood mononuclear cells from healthy donors $(n=5)$ in the presence of conventional or defucosylated monoclonal antibodies. Data are presented as the mean \pm standard deviation. ${ }^{*} \mathrm{P}<0.05$, as indicated. ADCC, antibody-dependent cellular cytotoxicity; MAPK, mitogen-activated protein kinase.

able to enhance the clinical efficacy of the therapeutic mAbs. For example, modification of antibodies to increase binding to $\mathrm{Fc} \gamma \mathrm{R}$ has been pursued in order to augment $\operatorname{ADCC}(11,12)$. It has finally been reported that removal of the $\alpha-1,6$ fucose moiety on the N-glycan at $\mathrm{Asn}^{297}$ of the heavy chain, which is called defucosylation technology, significantly enhanced ADCC in comparison to that of conventional antibody.
Previously, there is only one report describing the efficacy of defucosylated trastuzumab using PBMCs from breast cancer patients (16). Herein, our present study is the first report indicating usefulness of defucosylated cetuximab and trastuzumab for ADCC using the PBMCs of GI-tract cancer patients. Our observation and the previous report using clinical samples clearly confirmed the defucosylation technology efficiently can enhance the therapeutic mAbs-mediated ADCC.

In line with several previous reports $(23,33)$, we confirmed that conventional therapeutic mAbs-mediated ADCC in advanced disease was impaired in comparison to those in early disease or healthy donors. It is generally accepted that NK cells in cancer-bearing hosts are impaired by many mechanisms, including their reduced number, imbalances in their activating and inhibitory receptor, impaired activation signaling cascade as well as immunosuppressive cytokines $(8,23,24)$. However, even in such a condition, the current study clearly indicated that the defucosylated mAbs can restore the impaired ADCC in advanced diseases.

MAPK inhibitors have been originally developed for anti-cancer drugs based on their anti-proliferative action against tumor cells $(34,35)$. In addition, we and others previously reported that the MAPK inhibitor can induce up-regulation of HLA Class I and down-regulation of MICA/B expression on tumor cells, leading to less NK sensitivity $(26,27)$. Therefore, in the present study, we tried to mimic immunosuppressive status specific for NK-killing by modulating ADCC-related molecules with MAPK inhibitors. As a result, we confirmed that conventional cetuximab- and trastuzumab-mediated ADCC was impaired when target tumor cells were pre-treated with the MAPK inhibitor. Of importance, the defucosylated therapeutic mAbs can enhance ADCC activities even if the NKG2D ligand and MHC class I expression on tumor cells were modified by the MAPK inhibitor into immunosuppressive status. Taking it into consideration, the defucosylated therapeutic $\mathrm{mAbs}$ have a strong capability to enhance ADCC activities.

Theoretically, it would be likely that the defucosylated therapeutic mAbs has no ADCC activity against normal 
tissues, since the therapeutic potential of the mAbs are dependent on the level of target antigens expressed on the surface of target cells. When we consider the clinical application of the defucosylated therapeutic mAbs described in the present study, further study to exclude potential toxicities of the defucosylated mAbs to tissues expressing low levels of such target antigens will be needed.

In conclusion, the defucosylated therapeutic mAbs can restore the impaired ADCC activities in advanced stage of cancer patients, leading to more effective anti-cancer treatments.

\section{References}

1. Ferlay J, Soerjomataram I, Dikshit R, Eser S, Mathers C, Rebelo M, Parkin DM, Forman D and Bray F: Cancer incidence and mortality worldwide: Sources, methods and major patterns in GLOBOCAN 2012. Int J Cancer 136: E359-E386, 2015.

2. Bang YJ, Van Cutsem E, Feyereislova A, Chung HC, Shen L, Sawaki A, Lordick F, Ohtsu A, Omuro Y, Satoh T, et al: Trastuzumab in combination with chemotherapy versus chemotherapy alone for treatment of HER2-positive advanced gastric or gastro-oesophageal junction cancer (ToGA): A phase 3 , open-label, randomised controlled trial. Lancet 376: 687-697, 2010.

3. Li K and Li J: Current molecular targeted therapy in advanced gastric cancer: A comprehensive review of therapeutic mechanism, clinical trials, and practical application. Gastroenterol Res Pract 2016: 4105615, 2016

4. Arienti C, Zanoni M, Pignatta S, Del Rio A, Carloni S, Tebaldi M, Tedaldi G and Tesei A: Preclinical evidence of multiple mechanisms underlying trastuzumab resistance in gastric cancer. Oncotarget 7: 18424-18439, 2016.

5. Koene HR, Kleijer M, Algra J, Roos D, von dem Borne AE and de Haas M: Fc gammaRIIIa-158V/F polymorphism influences the binding of IgG by natural killer cell Fc gammaRIIIa, independently of the Fc gammaRIIIa-48L/R/H phenotype. Blood 90 : $1109-1114,1997$.

6. Shields RL, Namenuk AK, Hong K, Meng YG, Rae J, Briggs J, Xie D, Lai J, Stadlen A, Li B, et al: High resolution mapping of the binding site on human IgG1 for Fc gamma RI, Fc gamma RII, Fc gamma RIII, and FcRn and design of IgG1 variants with improved binding to the Fc gamma R. J Biol Chem 276: 6591-6604, 2001.

7. Warmerdam PA, van de Winkel JG, Vlug A, Westerdaal NA and Capel PJ: A single amino acid in the second Ig-like domain of the human Fc gamma receptor II is critical for human IgG2 binding. J Immunol 147: 1338-1343, 1991.

8. Watanabe M, Kono K, Kawaguchi Y, Mizukami Y, Mimura K, Maruyama T, Izawa S and Fujii H: NK cell dysfunction with down-regulated CD16 and up-regulated CD56 molecules in patients with esophageal squamous cell carcinoma. Dis Esophagus 23: 675-681, 2010.

9. Musolino A, Naldi N, Bortesi B, Pezzuolo D, Capelletti M, Missale G, Laccabue D, Zerbini A, Camisa R, Bisagni G, et al: Immunoglobulin $\mathrm{G}$ fragment $\mathrm{C}$ receptor polymorphisms and clinical efficacy of trastuzumab-based therapy in patients with HER-2/neu-positive metastatic breast cancer. J Clin Oncol 26: 1789-1796, 2008.

10. Wormald S, Milla L and O'Connor L: Association of candidate single nucleotide polymorphisms with somatic mutation of the epidermal growth factor receptor pathway. BMC Med Genomics 6: 43, 2013

11. Shields RL, Lai J, Keck R, O'Connell LY, Hong K, Meng YG, Weikert SH and Presta LG: Lack of fucose on human IgG1 N-linked oligosaccharide improves binding to human Fcgamma RIII and antibody-dependent cellular toxicity. J Biol Chem 277: 26733-26740, 2002

12. Shinkawa T, Nakamura K, Yamane N, Shoji-Hosaka E, Kanda Y, Sakukrada M, Uchida K, Anazawa H, Satoh M, Yamasaki M, et al: The absence of fucose but not the presence of galactose or bisecting $\mathrm{N}$-acetylglucosamine of human IgG1 complex-type oligosaccharides shows the critical role of enhancing antibody-dependent cellular cytotoxicity. J Biol Chem 278: 3466-3473, 2003.
13. Niwa R, Shoji-Hosaka E, Sakurada M, Shinkawa T, Uchida K, Nakamura K, Matsushima K, Ueda R, Hanai N and Shitara K: Defucosylated chimeric anti-CC chemokine receptor 4 IgG1 with enhanced antibody-dependent cellular cytotoxicity shows potent therapeutic activity to T-cell leukemia and lymphoma. Cancer Res 64: 2127-2133, 2004.

14. Okazaki A, Shoji-HosakaE, Nakamura K, Wakitani M, Uchida K, Kakita S, Tsumoto K, Kumagai I and Shitara K: Fucose depletion from human IgG1 oligosaccharide enhances binding enthalpy and association rate between IgG1 and FcgammaRIIIa. J Mol Biol 336: 1239-1249, 2004

15. Niwa R, Hatanaka S, Shoji-Hosaka E, Sakurada M, Kobayashi Y, Uehara A, Yokoi H, Nakamura K and Shitara K: Enhancement of the antibody-dependent cellular cytotoxicity of low-fucose IgG1 Is independent of FcgammaRIIIa functional polymorphism. Clin Cancer Res 10: 6248-6255, 2004.

16. Suzuki E, Niwa R, Saji S, Muta M, Hirose M, Iida S, Shiotsu Y, Satoh M, Shitara K, Kondo M and Toi M: A nonfucosylated anti-HER 2 antibody augments antibody-dependent cellular cytotoxicity in breast cancer patients. Clin Cancer Res 13: 1875-1882, 2007.

17. Capes-Davis A, Theodosopoulos G, Atkin I, Drexler HG, Kohara A MacLeod RA, Masters JR, Nakamura Y, Reid YA, Reddel RR and Freshney RI: Check your cultures! A list of cross-contaminated or misidentified cell lines. Int J Cancer 127: 1-8, 2010.

18. Shiraishi K, Mimura K, Izawa S, Inoue A, Shiba S, Maruyama T, Watanabe M, Kawaguchi Y, Inoue M, Fujii $\mathrm{H}$ and Kono $\mathrm{K}$ : Lapatinib acts on gastric cancer through both antiproliferative function and augmentation of trastuzumab-mediated antibody-dependent cellular cytotoxicity. Gastric Cancer 16: 571-580, 2013.

19. Carter P, Presta L, Gorman CM, Ridgway JB, Henner D, Wong WL, Rowland AM, Kotts C, Carverr ME and Shepard HM: Humanization of an anti-p185HER2 antibody for human cancer therapy. Proc Natl Acad Sci USA 89: 4285-4289, 1992.

20. DrugBANK. https://www.drugbank.ca/drugs/DB00002.

21. Yamane-Ohnuki N, KInoshita S, Inoue-Urakubo $M$, Kusunoki M, Iida S, Nakano R, Wakitani M, Niwa R, Sakurada M, Uchida K, et al: Establishment of FUT8 knockout Chinese hamster ovary cells: An ideal host cell line for producing completely defucosylated antibodies with enhanced antibody-dependent cellular cytotoxicity. Biotechnol Bioeng 87: 614-622, 2004

22. Mimura K, Kamiya T, Shiraishi K, Kua LF, Shabbir A, So J, Yong WP, Suzuki Y, Yoshimoto Y, Nakano T, et al: Therapeutic potential of highly cytotoxic natural killer cells for gastric cancer. Int J Cancer 135: 1390-1398, 2014.

23. Kono K, Takahashi A, Ichihara F, Sugai H, Fujii H and Matsumoto Y: Impaired antibody-dependent cellular cytotoxicity mediated by herceptin in patients with gastric cancer. Cancer Res 62: 5813-5817, 2002.

24. Mimura K, Kono K, Hanawa M, Kanzaki M, Nakao A, Ooi A and Fujii H: Trastuzumab-mediated antibody-dependent cellular cytotoxicity against esophageal squamous cell carcinoma. Clin Cancer Res 11: 4898-4904, 2005.

25. Moretta L, Locatelli F, Pende D, Marcenaro E, Mingari MC and Moretta A: Killer Ig-like receptor-mediated control of natural killer cell alloreactivity in haploidentical hematopoietic stem cell transplantation. Blood 117: 764-771, 2011.

26. Bae DS, Hwang YK and Lee JK: Importance of NKG2D-NKG2D ligands interaction for cytolytic activity of natural killer cell. Cell Immunol 276: 122-127, 2012.

27. Okita R, Mougiakakos D, Ando T, Mao Y, Sarhan D, Wennerberg E, Seliger B, Lundqvist A, Mimura K and Kiessling R: HER2/HER3 signaling regulates NK cell-mediated cytotoxicity via MHC class I chain-related molecule A and B expression in human breast cancer cell lines. J Immunol 188: 2136-2145, 2012

28. Watanabe $\mathrm{Y}$, Asano $\mathrm{R}$, Arai $\mathrm{K}$, Shimomura I, Ogata $\mathrm{H}$, Kawaguchi $\mathrm{H}$, Hayashi $\mathrm{H}$, Ohtsuka $\mathrm{H}$, Yoshida $\mathrm{H}$, Katayose Y, et al: In vitro and in vivo antitumor effects of recombinant bispecific antibodies based on humanized anti-EGFR antibody. Oncol Rep 26: 949-955, 2011.

29. Baselga J, Carbonell X, Castañeda-Soto NJ, Clemens M, Green M, Harvey V, Morales S, Barton C and Ghahramani P: Phase II study of efficacy, safety, and pharmacokinetics of trastuzumab monotherapy administered on a 3-weekly schedule. J Clin Oncol 23: 2162-2171, 2005.

30. Hudis CA: Trastuzumab-mechanism of action and use in clinical practice. N Engl J Med 357: 39-51, 2007. 
31. Slamon DJ, Leyland-Jones B, Shak S, Fuchs H, Paton V, Bajamonde A, Fleming T, Eiermann W, Wolter J, Pegram M, et al: Use of chemotherapy plus a monoclonal antibody against HER2 for metastatic breast cancer that overexpresses HER2. N Engl J Med 344: 783-792, 2001

32. Kawaguchi Y, Kono K, Mimura K, Mitsui F, Sugai H, Akaike H and Fujii H: Targeting EGFR and HER-2 with cetuximab- and trastuzumab-mediated immunotherapy in oesophageal squamous cell carcinoma. Br J Cancer 97: 494-501, 2007.

33. Kawaguchi Y, Kono K, Mimura K, Sugai H, Akaike H and Fujii H: Cetuximab induce antibody-dependent cellular cytotoxicity against EGFR-expressing esophageal squamous cell carcinoma. Int J Cancer 120: 781-787, 2007.
34. Omori S, Hida M, Fujita H, Takahashi H, Tanimura S, Kohno M and Awazu M: Extracellular signal-regulated kinase inhibition slows disease progression in mice with polycystic kidney disease. J Am Soc Nephrol 17: 1604-1614, 2006.

35. Fujiwara Y, Hosokawa Y, Watanabe K, Tanimura S, Ozaki K and Kohno M: Blockade of the phosphatidylinositol-3-kinase-Akt signaling pathway enhances the induction of apoptosis by microtubule-destabilizing agents in tumor cells in which the pathway is constitutively activated. Mol Cancer Ther 6: 1133-1142, 2007. 